\title{
SARS-CoV-2 spike and Telomerase RNAs compared to arrive at an explanation for increased ageing in alveolar cells in severe COVID-19
}

\author{
Han Geurdes ${ }^{1}$
}

GDS Appl Math BV Den Haag/Doetinchem, The Netherlands

\begin{abstract}
In this letter we investigate if SARS-CoV-2 RNA is involved in the increased ageing of alveolar cells. Our in silico study is explorative. With the results we are able to outline experiments with AEC2 repair of bleomycin damaged alveolar cells. If AEC2 repair capability is diminished by spike RNA then perhaps this result provides a first step on a route to treat immortal lung cancer cells.
\end{abstract}

\section{Introduction}

In a recent study of COVID-19, a (statistical) relation was found between severity of COVID-19 illness and a decrease in length of peripheral blood lymphocyte telomeres ${ }^{1}$. COVID-19 is caused by the SARS-CoV-2 virus which introduces its single strand RNA via the ACE2 and TMPRSS2 receptors into the cell ${ }^{2}$. SARS-CoV- 2 causes from mild flu-like symptoms in approximately $80 \%$ of the cases to a severe lung and multi-organic failure which can result in death of a significant percentage of patients (viz. Sanchez-Vazquez, 1).

Telomeres are chromosome ends to protect against rearrangement and the DNA broken strand repair system ${ }^{3}$ of the cell. When cells divide and DNA is replicated, the telomeres become shorter ${ }^{4}$. This is a normal consequence of cellular division and is a molecular mechanism of cellular ageing ${ }^{5}$.

Here we will focus on alveolar epithelial cells (AEC) and note a certain parallelism between the effect of severe COVID-19 on the lungs and idiopathic pulmonary fibrosis (IPF). The first thing we may observe is that lung alveolar integrity is related to telomere length ${ }^{6}$ and the activity of the enzyme telomerase. If the number of telomeres goes below the Hayflick limit, the cell enters the senescence and mortality stage ${ }^{7}$.

Telomerase is a ribonucleoprotein complex to maintain telomeres ${ }^{8}$. Telomerase is synthesized in stem / progenitor cells but its de novo synthesis in ordinary cells is suppressed. When an ordinary cell escapes mortality it becomes a cancer cell. Secondly, IPF is an illness with increased prevalence in advanced age with the hallmark of activation of AEC and epithelium driven accumulation of lung connective tissue ${ }^{9}$. The age factor suggests a role for telomeres and telomerase. Telomeres are therefore center stage here. Note e.g. also in early life, length of telomeres is dynamic ${ }^{10}$ and e.g. telomere reduction in skin cells is caused by UV radiation ${ }^{11}$.

\footnotetext{
${ }^{1}$ The author wishes to thank A. Popper, MSc for his stimulating comment and devotion to the in silico experiment.
} 
Thirdly there are two kinds of $A E C$. AEC1, responsible for oxygen processing and the progenitor cells $A E C 2$. The latter produce surfactant and can transform, when necessary, into AEC $1^{12}$. The ability to go from AEC2 to AEC1 is in need of telomerase (viz. Parra, 10). We note that human AEC2 has ACE2 receptors so AEC2 is vulnerable to SARS-CoV-2 infection (viz. Sanchez-Vazquez, 1).

Telomerase is a reverse transcriptase. The RNA (hTR) of telomerase is an integral part of the enzyme and contains the template to telomeres it attaches processively at the $3^{\prime}$ end of chromosomal DNA. The architecture of hTR in the complex ${ }^{13}$ disables hijacking by alien RNA. Furthermore, there is a control mechanism for the incorporation of RNA in telomerase ${ }^{14}$. When a lot of similar to hTR alien RNA is present in the cell, the assembly of telomerase might be hampered. When such an AEC2 cell later turns into AEC1, it will have less telomere repair possibilities and have shorter telomeres.

\section{Method}

Here, ways to compare RNA sequences are designed to establish a distance measure for "similar". In this step the secondary structure of the hTR is of importance (viz. Zhang, 13). We developed in our computational lab, a method based on multidimensional scaling descriptive statistics ${ }^{15,16}$ of similarities among objects. The basis of the scaling approach is a measure of similarity between object $i$ and object $j$, in symbols, $\delta_{i, j}$. The elementary objects here are the (NTs) nucleotides; $A, U(T), C$ and $G$. In RNA the NTs are connected by ribose phosphor sugar repetitive elements. In the present analysis we will only look at the sequences containing the NTs to determine $\delta_{i, j}$. Each NT at a certain position contribute to the $\delta_{i, j}$.

The first characteristic is pairwise comparison of quantum Helium approximate wave function $\Psi$ solutions of, $H \Psi=E \Psi$, and $H$ the Hamiltonian. I.e.

$$
\left\{-\frac{1}{2} \Delta_{1}-\frac{1}{2} \Delta_{2}+\frac{1}{r_{1,2}}-\frac{1}{r_{1}}-\frac{1}{r_{2}}\right\} \Psi=E \Psi
$$

Each atom pair in the NT molecule is treated as though their outer electrons are in a Helium "atom" with Hamiltonian as given (viz. Geurdes 16). In the potential energy term, $\frac{1}{r_{1,2}}-\frac{1}{r_{1}}-\frac{1}{r_{2}}$, the $r_{12}$ represent the distance between the two electrons and the $r_{k}$ with $k=1,2$, the distance between the electron and the (to those two) positive charged plane of the NT molecule. The E is the eigenvalue (energy) and the nabla's $-\frac{1}{2} \Delta_{k}$ represent the quantum kinetic energy in the He Hamiltonian. Mass and Planck constant are in unity units. Use is made of Hückel coefficients to identify the different atoms in the NT molecule. Later computations can e.g. try to introduce the LCAO molecular orbital method ${ }^{17}$ in determining the $\delta_{i, j}$.

A further qualitative (dis)similarity is based on $\mathrm{H}$ bridges between the NTs. This concept is also employed to determine a matrix for second order configuration influence. The second order structure matrix is believed to hold the (pseudo)knots that are relevant to the architecture of the RNA in the telomerase complex. Obviously, the complementarity computations in the matrix overestimate the architectural form. However, the biochemical relevant architecture is present as a sub matrix. 
In addition, per three NTs a four dimensional Euclidean distance computation was performed. Each NT represents a dimension in 4 space. Another point was a scaled qualitative categorization of similarity of three NTs in their amino acid effect. Finally, data from ATR-IR spectra (pubChem) were used for the 4 NTs to establish a similarity and to connect to a more semi-empiric set of data. Here axes of different NTs had to be transformed into each other in order to make the comparison. A normalization of $\left(\frac{1}{1+|n-m|}\right)$ was employed for the $\mathrm{n}$-th and $\mathrm{m}$-th NT in the two to be compared sequences.

Finally, RNA sequences are compared modulo the implicit restrictions and theoretical assumptions. The modeling details can be found in Geurdes, 16. In the present case we first employed classical MDS and subsequently isoMDS in R. Then the second order configuration matrix was employed to the two axes and a subsequent isoMDS provides the result projection in a two dimensional space.

In this space the $75 \%$ Euclidean radius of the circle around the origin $(0,0)$, i.e. , R75(00), is a measure of similarity of the two RNA sequences. $75 \%$ of the points lie within the perimeter. Its rationale is that the coordinates sum to $(0,0)$ in the projection. Furthermore, for computational convenience, the comparison in Fig. 1 is based upon 4 separate comparisons, each of size 271, with start points 1, 100, 199 and 268. Here, rotational freedom around the "out of plane" axis through $(0,0)$ is employed to obtain the configuration with the smallest R75(00).

\section{Material}

In the in silico experiments we employed S spike data from GenBank: MT419837.1 and GenBank: U86046.1 for hTR. 


\section{Results}

In Fig. 1 the results of computation are displayed. The comparison occurs: $5^{\prime}->3^{\prime}$. Only in (E) we have S2 $5^{\prime}->3^{\prime}$ vs hTR $3^{\prime}->5^{\prime}$.

(A) S2-hTR

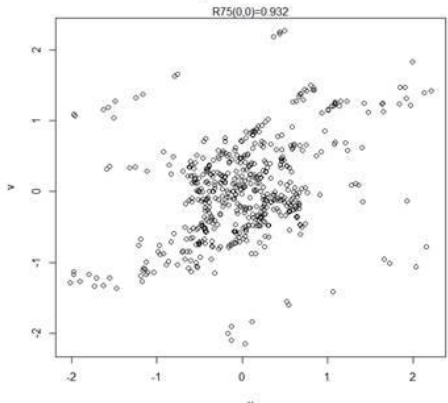

(C) S1-hTR

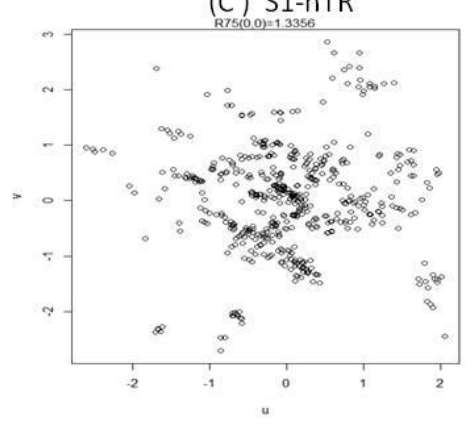

(B) S2-ranRNA

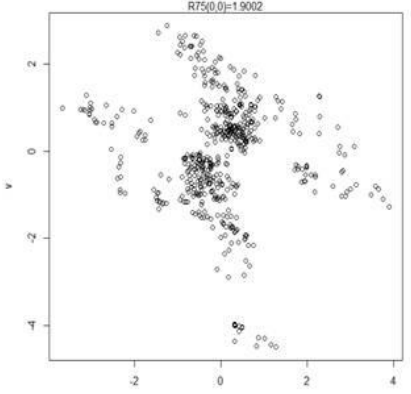

(D) S1-ranRNA

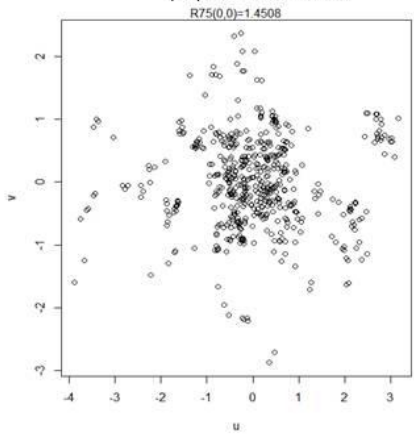

(E) S2-hTR 3'-5'

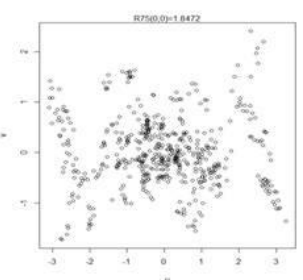

Figure 1 Projection in 2 dim space of S1 spike or S2 spike RNA vs hTR. (A) comparison of hTR with S2 spike; R75(0,0)=0.932. (B) S2 spike with randomly generated RNA; $R 75(0,0)=1.9002$. (C) S1 spike and $h T R, R 75(0,0)=1.3356$ (D) S1 versus random RNA, R75(0,0)=1.4508. (E) S2 spike 5' $->3^{\prime}$ matched with $3^{\prime}-5^{\prime}$ hTR, R75(0,0)=1.8472.

\section{Discussion}

Before presenting the result of our computations, an alternative explanation is given. Fibrosis can be caused by ACE2 blocking ${ }^{18}$. If the merging of the virus with the AEC2 membrane destroys the ACE2 enzyme, it most likely will induce multiple divisions in order to maintain the ACE2 enzyme function at a required level. Shortening of telomeres are expected in that case. Below we will explain telomere shortening in severe COVID-19 as follows.

With the use of a 2 dimensional projection, the match with randomly generated RNA, Fig. 1 (B) \& (D), for both S1 and S2 produces a larger R75 $(0,0)$ than with biological RNA. Further, S2 has the lowest R75 $(0,0)$ value. Moreover, reversal of the direction of comparing $5^{\prime}->3^{\prime}$ S2 RNA with $3^{\prime}->5^{\prime}$ hTR gave a relatively high $R 75(0,0)$, viz. Fig. $1(E)$ vs $(A)$. Modulo the assumptions in our model, we then $b$ can conclude that our in silico experiments indicate that the S2 part of the SARS-CoV-2 spike mRNA (start at RNA position: $21563+2028=23591$ is 1 S2 RNA) best fit the hTR of telomerase. Therefore, the in vitro experiment that we propose is to have AEC2 cells that are able to assemble telomerase and to introduce in those cells SARS-CoV-2 S2 spike RNA.

A possible experimental set up is in vitro bleomycin-induced lung epithelial cell (LEC) apoptosis. Let us concentrate on AEC2 cells. Bleomycin causes an initial increase, and then a reduction, in telomerase activity... ${ }^{19}$. The influence of S2 spike RNA on telomerase synthesis can therefore be quantified by looking at the first $24 \mathrm{~h}$ telomerase increase in LEC of the ARC2 kind (viz. 
Fridlender, 19). We predict that S2 spike RNA treated AEC2 will produce less telomerase activity in this peak of $24 \mathrm{~h}$. The reduction in the activity can be quantified with G-quadruplex-intercalating porphyrin telomerase inhibitor. Further, the telomerase activity can also be quantified with PCR based telomere repeat amplification protocol (TRAP) ${ }^{20}$. Detection of telomerase activity is briefly presented in reference 19. An in vivo experiment can be modeled as an AEC2 transplantation experiment ${ }^{21}$ where $A E C 2$ and S2 RNA treated AEC2 can give a difference in recovery grade from bleomycine induced lung injury.

Finally we note that if the S2 RNA lowers the telomerase activity then a first step on the road to a possible treatment of proliferation of immortal lung cells could be found. Telomerase activity was found to be absent in most normal human somatic cells but present in over $90 \%$ of cancerous cells (viz. Cong, 7). Immortalization is the hallmark of malignant transformation and a premalignant phenotype $^{22}$. If S2 RNA has the predicted hampering effect on telomerase synthesis and can be delivered to the cancer cells, then the immortality of this type of cells is destroyed. Perhaps that the increase of caveolin-1 secretion of immortalized AEC2, can be a sign for a possible parasite vector delivering the S2 RNA.

\section{References}

\footnotetext{
${ }^{1}$ Sanchez-Vazquez, R. et al. Shorter telomere lengths in patients with severe COVID-19 disease, Ageing 2021, 13:1.

${ }^{2}$ Wan, Y. et al. Receptor Recognition by the Novel Coronavirus from Wuhan: an Analysis Based on DecadeLong Structural Studies of SARS Coronavirus, Journal of Virology 2020, 94:e00127.

${ }^{3} \mathrm{Wu}, \mathrm{R}$. et al. Telomerase mechanism of telomere synthesis, Annu Rev Biochem 2017, 86:439.

${ }^{4}$ Martinez, P. and Blasco, M. Telomeric and extra-telomeric roles for telomerase and teleomere-binding proteins, Nat, Rev Cancer, 2011, 11:116.

${ }^{5} \mathrm{Mir}, \mathrm{S}$. et al. Shelterin complex at telomeres: implications in ageing, Clin. Interv. Ageing, 2020, 15:827.

${ }^{6}$ Lee, J. et al. Lung alveolar integrity is compromised by telomere shortening in telomerase-null mice, 2009,296:L57.

${ }^{7}$ Cong, Y. et al. Human telomerase and its regulation, Microbiol, Molecularbiol Rev. 2002, 66:407.

${ }^{8}$ Chen, R. et al. Telomerase deficiency causes alveolar stem cell senescence associated low grade inflammation in lungs, Cell, 2015, 290:30813.

9 Pardo, A. et al. Lung Fibroblasts, ageing and Idiopathic pulmonary fibrosis, Ann. Am. Thoracic Soc, 2016, 13:S417.

${ }^{10}$ Bertucci, E. et al. The ageing DNA methylome reveals environment-by-ageing interactions in a model teleost, 2021, biorXiv, 10.1101/2021.03.01.433371.

${ }^{11}$ Stout, G. et al. Telomere length and telomerase activity impact the UV sensitivity syndrome Xeroderma Pigmentosum, Mol and Cell Pathol, 2013, 73:1844.

12 Parra, E. et al. Modeling pulmonary fibrosis by abnormal expression of telomerase/apoptosis/collagen $V$ in experimental usual interstitial pneumonia, Brazilian Journal of Medical and Biological Research, 2014, 47:567.

${ }^{13}$ Zhang, Q. et al. Architecture of human telomerase RNA, PNAS 2011, 51:20325 (see fig.1 in this publication).

${ }^{14} \mathrm{Hu}$, X. et al. Quality-Control Mechanism for Telomerase RNA Folding in the Cell, Cell Reports, 2020, 33:108568.

15 a Cox, T. \& Cox, M. Multidimensional scaling $2^{\text {nd }}$ edition, Chapman 2001, Boca Raton.

${ }^{\text {b}}$ Ripley, B. Pattern recognition and neural networks, Cambridge Univ. Press, 1996, Cambridge, UK.

${ }^{16}$ Geurdes, H. Approximative He Hamiltonian in descriptive multidimensional scaling statistics of RNA contained information with application to SARS-CoV-2 S spike RNA and 7SLRNA, 2021, https://ssrn.com/abstract $=3772670$ \& under review.

${ }^{17}$ Lowe, J. Quantum chemistry, 1978, Elsevier (chap 11).

${ }^{18}$ Verdechia, R. et al. The pivotal link between ACE2 deficiency and SARS-CoV-2 infection, Eur J Intern Med, 2020, 76:14.

${ }^{19}$ Fridlender, Z. et al. Telomerase activity in bleomycin-induced epithelial cell apoptosis and lung fibrosis, Eur Respr J, 2007, 30:205.
} 
${ }^{20}$ Bryan, M. et al. Telomere length dynamics in telomerase positive immortal human cell populations, Exp. Cell Res, 1998, 239:370.

${ }^{21}$ Alvarez-Palomo, B. et al. Induced pluripotent stem cell-derived lung alveolar epithelial type II cells reduce damage in bleomycin-induced lung fibrosis, Stem Cell Research \& Therapy, 2020, 11:213.

22 Milyavsky, M. et al. Prolonged culture of telomerase - immortalized human fibroblasts leads to a premalignant phenotype, Canc. Res. 2003,63:7147. 